\title{
Impact of CA19-9 in Diagnosis of Combined Hepatocellular Carcinoma and Cholangiocarcinoma
}

\author{
Y.S.Younes $^{1}$, B.A.Abdul-Aziz ${ }^{1}$, W.A.El-Agawy ${ }^{2}$ and W.M.M.Ahmed ${ }^{1}$ \\ ${ }^{1}$ Hepatology, Gastroenterology \& Infectious Diseases Dept., Faculty of Medicine, Benha Univ., Benha, Egypt \\ ${ }^{2}$ Tropical Medicine Dept., Faculty of Medicine, Benha Univ., Benha, Egypt \\ E-Mail:W.M56@gmail.com
}

\begin{abstract}
Hepatocellular carcinoma (HCC) is the sixth most common cancer worldwide. Intrahepatic cholangiocarcinoma (ICC) accounts for about 10-15\% of all primary hepatic malignancy. Combined hepatocellular carcinoma and cholangiocarcinomas (cHCC-CCs) are rare malignancies representing 1.0-4.7\% of all primary liver cancers. ICC and cHCC-CC preoperative diagnosis is desirable because frequency of lymph node metastasis. Also they have been considered a contraindication for liver transplantation (LT).The study aimed to assess the importance of CA19-9 in diagnosis of cHCC-CC "non-invasively" in patients with typical radiological features of HCC which may alter the interventional procedure. This observational cross-sectional study was conducted on 62 patients with typical radiological criteria of HCC and elevated CA19-9 level, who attended to National Hepatology and Tropical Medicine Research Institute (NHTMRI); Cairo, Benha university hospitals within the period between October 2017 and April 2019. All studied subjects underwent history taking, clinical examination, biochemical workup, tumor markers, abdominal ultrasound, and dynamic study (CT/MRI). Target liver biopsy was done for histopathology and immuno-histochemistry. The studied patients were classified into three groups based on pathological diagnosis. 20 patients were diagnosed as HCC, 40 patients were diagnosed as ICC, and 2 patients were diagnosed as cHCC-CC with statistically significant difference between HCC and ICC as regard CA19-9 and alpha-fetoprotein (AFP). CA19-9 and AFP cut offs were $>64.8 \mathrm{U} / \mathrm{mL}$ and $<23.6 \mathrm{ng} / \mathrm{mL}$, respectively favoring the diagnosis of ICC. CA19-9 level was 176.3 and $156.7 \mathrm{U} / \mathrm{mL}$ while AFP level was 460 and $170 \mathrm{ng} / \mathrm{mL}$ in cHCC-CC cases, respectively. The use of CA19-9 can help in diagnosis of ICC and suspicion of cHCC-CC in cases of typical radiological criteria of HCC.
\end{abstract}

Keywords Hepatocellular carcinoma, Intrahepatic cholangiocarcinoma, Combined hepatocellular carcinoma and cholangiocarcinoma, Alpha-fetoprotein, Carbohydrate Antigen 19-9.

\section{Introduction}

HCC represents the sixth most common cancer worldwide.1 In Egypt; it represents the fourth common cancer. 2

ICC is one of the subtypes of cholangiocarcinoma, which accounts for about 10$15 \%$ of all primary hepatic malignancies. 3

Misdiagnosis of ICC as HCC may result in selection of a treatment option (such as TACE or RFA) that is effective for HCC but not as effective for CCA. 4

ICCs are associated with poor outcomes. ICCs considered a contraindication for LT.5

cHCC-CC is a rare primary liver cancer containing elements of both HCC and ICC and accounting for $1.0-4.7 \%$ of all primary liver cancers. 6

The presence of imaging features of both HCC and CCA in the same tumor may alert the radiologist to the possibility of $\mathrm{cHCC}-\mathrm{CC}$ though.7 But the definite diagnosis of cHCC-CC can be made by histopathological examination only with use of Immuno-histochemistry (IHC). 8

Correct preoperative diagnosis is desirable because the high frequency of lymph node metastasis, making its dissection a necessity for curative resection. 9

LT has no role and is not a good management option for cHCC-CC.10 Outcomes are worse when compared with HCC due to associated higher recurrence rates after transplant. 11
So, this study was conducted aiming to assess the significance of elevated CA19-9 in suspecting diagnosis of cHCC-CC "non-invasively" in patients with typical radiological features of HCC which may alter the interventional procedure.

\section{Patients and methods}

This observational study was conducted on 62 patients with radiological criteria of HCC and elevated CA19-9 level, who attended to National Hepatology and Tropical Medicine Research Institute (NHTMRI); Cairo, Benha university hospitals within the period between October 2017 and April 2019.

They were divided into three groups: Group (1) included 20 patients diagnosed as HCC, Group (2) included 40 patients diagnosed as ICC and Group (3) included two patients diagnosed as $\mathrm{CHCC}-\mathrm{CC}$.

All patients were underwent history taking, clinical examination, detailed biochemical investigations (complete blood count, bilirubin level (T,D), serum albumin, INR and renal functions), viral markers, tumor markers (AFP and CA19-9), abdominal ultrasound, and dynamic study.

Liver biopsy was done using 18-gauge core biopsy needle and the collected liver tissue was put into formalin immediately and the blocks were prepared from the collected tissue specimen and the sections were stained with Hematoxylin and Eosin (H\&E).

Sections were treated by using specific monoclonal antibodies against GPC3, Hep-par1, CK7 
and CK19. Chromogen and hematoxylin was applied as counter-stain for IHC staining.

\subsection{Ethical approved}

This work was approved by the scientific committee of Benha University Hospitals. All included patients were informed about the procedure of the study and gave a written informed consent to participate in the study.

\subsection{Statistical analysis}

Patients' data were analyzed using Statistical Program for Social Science (SPSS) version 15.0 for windows. Quantitative data were expressed as mean \pm standard deviation (SD) and median. Qualitative data were expressed as frequency (No.) and percentage (\%). Chi-square test (X2) was used when comparing between non-parametric data. P-value was set at $\leq 0.05$ for significant results and $>0.05$ for insignificant results. ROC curve was used to determine cut off value and the following statistics can be defined: sensitivity, specificity, positive predictive value (PPV) and negative predictive value (NPV).

\section{Results}

The 62 patients were chosen from 622 patients with typical radiological criteria of $\mathrm{HCC}$, for them CA19-9 was done and who showed elevated level was included in this study.

The studied patients were classified into three groups based on pathological diagnosis. 20 patients were diagnosed as HCC, 40 patients were diagnosed as ICC, and 2 patients were diagnosed as cHCC-CC.

Two patients were diagnosed as $\mathrm{cHCC}-\mathrm{CC}$ with an incidence $0.32 \%$ from all primary liver tumors patients visited the NHTMRI during the study period.

They were 58 and 45 years old males; none of them showed signs of liver cell failure during clinical examination and laboratory assessment. Both cases serology was positive for $\mathrm{HCV}$ and negative for HBV.

AFP level was 460 and $170 \mathrm{ng} / \mathrm{mL}$, and CA19-9 level was 176.3 and $156.7 \mathrm{U} / \mathrm{mL}$ for both cases, respectively.

For both cases liver biopsy was done and histopathological result was HCC grade II with mucin production was noted. On IHC, malignant cells were positive for GPC3, Hep-par1, CK7 and CK19; these results were consistent with $\mathrm{cHCC}-\mathrm{CC}$.

As regard the other groups; the mean age was $63.27 \pm 8.76$ years in HCC and $59.60 \pm 9.79$ years in ICC patients, and $63.6 \%$ of HCC and $73.3 \%$ of ICC patients were males, with no statistically significant difference ( $P$-value was 0.16 and 0.45 , respectively).

All patients of both groups complained of significant weight loss, none of HCC and $26.75 \%$ of ICC patients had jaundice, $63.6 \%$ of $\mathrm{HCC}$ and only $6.7 \%$ of ICC patients had esophageal varices, with statistically significant difference $(P$-value was 0.008 and 0.001 , respectively).

As regard laboratory findings; the mean $\mathrm{Hb}$ level was $11.5 \pm 1.87 \mathrm{gm} / \mathrm{dL}$ in HCC and $11.61 \pm 1.53 \mathrm{gm} / \mathrm{dL}$ in ICC, the mean WBCs count was 7.23 \pm 4.12 thousands/Cmm in $\mathrm{HCC}$ and $7.78 \pm 4.18$ thousands/Cmm in ICC, the median ALT was $45 \mathrm{U} / \mathrm{L}$ in $\mathrm{HCC}$ and $47.5 \mathrm{U} / \mathrm{L}$ in ICC, the mean serum albumin was $3.61 \pm 0.44 \mathrm{gm} / \mathrm{dL}$ in $\mathrm{HCC}$ and $3.43 \pm 0.57$ $\mathrm{gm} / \mathrm{dL}$ in ICC, the mean total bilirubin was $1.56 \pm 0.37$ $\mathrm{mg} / \mathrm{dL}$ in HCC and $1.35 \pm 0.71 \mathrm{mg} / \mathrm{dL}$ in ICC, the mean INR was $1.2 \pm 0.17$ in HCC and $1.18 \pm 0.23$ in ICC, the mean serum creatinine was $0.86 \pm 0.31$ $\mathrm{mg} / \mathrm{dL}$ in $\mathrm{HCC}$ and $1.22 \pm 0.31 \mathrm{mg} / \mathrm{dL}$ in ICC, with no statistically significant difference $(P$-value was 0.8 , $0.89,0.92,0.12,0.24,0.37$ and 0.6 , respectively).

As regard the mean platelets count was $165.55 \pm 78.09$ thousands/Cmm in $\mathrm{HCC}$ and $280.87 \pm 105.57$ thousands/Cmm in ICC patients, with statistically significant difference $(P$-value was $<0.001$ ).

As regard tumor markers; the median AFP was $46.3 \mathrm{ng} / \mathrm{mL}$ in $\mathrm{HCC}$ and $8.7 \mathrm{ng} / \mathrm{mL}$ in ICC patients, while the median CA19-9 was $67.4 \mathrm{U} / \mathrm{mL}$ in $\mathrm{HCC}$ and $155.7 \mathrm{U} / \mathrm{mL}$ in ICC patients, with statistically significant difference ( $P$-value was 0.002 and 0.003 , respectively).

As regard histopathological results of $\mathrm{HCC}$ patients; $63.6 \%$ were HCC grade I, $27.3 \%$ were HCC grade II and $9.1 \%$ were HCC grade III. And as regard ICC patients; $6.7 \%$ were moderately differentiated adenocarcinoma, $60 \%$ were poorly differentiated adenocarcinoma and $33.3 \%$ were undifferentiated malignant tumor.

As regard IHC; all cases of HCC showed positive staining for GPC3 and Hep-par1 and negative staining for CK7 and CK19, while all cases of ICC showed positive staining for CK7 and CK19 and negative staining for GPC3, and Hep-par1, there was statistically significant difference $(P$-value was $<0.001)$.

Using ROC curve, it was shown that CA19-9 can be used in discrimination between HCC and ICC patients at a cut off level of $>64.8 \mathrm{U} / \mathrm{mL}$, with $95.2 \%$ sensitivity, $57.4 \%$ specificity, $71.1 \% \quad \mathrm{PPV}$ and $87.06 \%$ NPV ( $P$-value was 0.002$)$.

Using ROC curve, it was shown that AFP can be used in discrimination between $\mathrm{HCC}$ and ICC patients at a cut off level of $<23.6 \mathrm{ng} / \mathrm{mL}$, with $88.1 \%$ sensitivity, $61.8 \%$ specificity, $71.11 \%$ PPV and $79.6 \%$ NPV ( $P$-value was 0.002$)$.

\section{Discussion}

This study was conducted on 62 patients with typical radiological criteria of $\mathrm{HCC}$ and elevated CA19-9 aiming to evaluate its significance in suspecting $\mathrm{cHCC}-\mathrm{CC}$.

Two cases were diagnosed as $\mathrm{cHCC}-\mathrm{CC}$ with an incidence $0.32 \%$ from all primary liver tumors, and this was in agreement with Wang et al. ${ }^{12}$ who 
reported combined HCC-CC incidence accounts for $0.4-14.2 \%$ of primary liver cancer cases.

AFP level was 460 and $170 \mathrm{ng} / \mathrm{mL}$, and CA19-9 level was 176.3 and $156.7 \mathrm{U} / \mathrm{mL}$ for both cases, and this was in agreement with Sciarra et al. ${ }^{13}$ who reported the combination of elevated CA19-9 and AFP should alert investigators for the possibility of cHCC-CC

For both cases a target liver biopsy was done and histopathological result was HCC grade II with mucin production was noted, and this was in agreement with IARC WHO $4^{\text {th }}$ edition. $^{14}$ who reported differentiated hepatocellular and biliary components in the tumor is required for histopathological diagnosis of cHCC-CC.

On IHC, malignant cells were positive for GPC3, Hep-par1, CK7 and CK19; these results were consistent with $\mathrm{cHCC}-\mathrm{CC}$, and this was in agreement with Tickoo et al. ${ }^{15}$ who reported the same IHC for both malignant components in cases of cHCC-CC.

As regard the other groups the mean age was

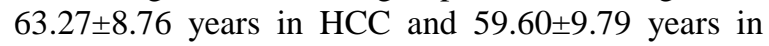
ICC patients, with no statistically significant difference between the two groups, and this was in agreement with Jung et al. ${ }^{16}$ who reported that mean age were no different between two groups.

In the current study $63.6 \%$ of HCC and $73.3 \%$ of ICC patients were males, with no statistically significant difference between the two groups, and this was in agreement with Wakizaka et al. ${ }^{17}$ who reported the majority of HCC and ICC cases occur in men.

Esophageal varices were more common in $\mathrm{HCC}$ than ICC patients $(63.6 \%$ and only $6.7 \%$, respectively), with statistically significant difference between the two groups, and this was in agreement with Wakizaka et al. ${ }^{17}$ and Lee et al. ${ }^{18}$ who reported that esophageal varices and liver cirrhosis were predominant in HCC patients.

Jaundice was predominant in ICC $(26.75 \%)$ than HCC patients, with statistically significant difference between the two groups, and this was in agreement with $\mathrm{Li}$ et al. $^{19}$ who reported predominance of jaundice in ICC patients.

PLT count was lower in HCC than ICC patients (mean was 165.55 \pm 78.09 and 280.87 \pm 105.57 thousands/Cmm, respectively), there was statistically significant difference between the two groups, and this was in agreement with Aljumah et al. ${ }^{20}$ who reported mild thrombocytopenia in HCC patients, and Gigante et al. ${ }^{21}$ who reported normal PLT count to mild thrombocytosis in ICC patients.

In this study AFP was higher in HCC than ICC patients (median was 46.3 and $8.7 \mathrm{ng} / \mathrm{mL}$, respectively), there was statistically significant difference between the two groups, and this was in agreement with Hsieh et al. ${ }^{22}$ who reported the median AFP was $59.6 \mathrm{ng} / \mathrm{mL}$ in HCC patients, and Gigante et al. ${ }^{21}$ who reported the median AFP was 9.1 $\mathrm{ng} / \mathrm{mL}$ in ICC patients.
But CA19-9 was higher in ICC than HCC patients (median was 155.7 and $67.4 \mathrm{U} / \mathrm{mL}$, respectively), there was statistically significant difference between the two groups, and this was in agreement with Lee et al. $^{23}$ who reported the median CA19-9 was 56.1 $\mathrm{U} / \mathrm{mL}$ in HCC patients, and Lumachi et al. ${ }^{24}$ who reported the median CA19-9 was $170.5 \mathrm{U} / \mathrm{mL}$ in ICC patients.

Histopathological results in HCC patients; $63.6 \%$ were HCC grade I, $27.3 \%$ were HCC grade II and $9.1 \%$ were HCC grade III, and this was in agreement with Zhang et al. $^{25}$ who reported the same pathological grading in HCC cases.

But histopathological results of ICC patients; $6.7 \%$ were moderately differentiated adenocarcinoma, $60 \%$ were poorly differentiated adenocarcinoma and $33.3 \%$ were undifferentiated malignant tumor, and this was in agreement with Sapisochin et al. $^{26}$ who reported the same pathological differentiation in ICC patients.

As regard IHC; all HCC cases showed positive staining for GPC3 and Hep-par1 and negative staining for CK7 and CK19, while ICC cases showed positive staining for CK7 and CK19 and negative staining for GPC3, and Hep-par1, with statistically significant difference between the two groups, and this was in agreement with Ryu et al. ${ }^{27}$ comparative study who reported the same results and differences between HCC and ICC cases.

Using ROC curve analysis revealed that CA19-9 can be used in discrimination between HCC and ICC patients at a cut off level of $>64.8 \mathrm{U} / \mathrm{mL}$, with $95.2 \%$ sensitivity, $57.4 \%$ specificity, $71.1 \%$ PPV and $87.06 \%$ NPV; area under the curve was 0.77 and Pvalue was 0.002 denoting good predicative value of CA19-9 in prediction of ICC, and this was in agreement with Li et al. ${ }^{28}$ who reported a CA19-9 cut off level of $>125.07 \mathrm{U} / \mathrm{mL}$, with $76.67 \%$ sensitivity and $80 \%$ specificity for diagnosis of CCA.

Using ROC curve analysis revealed that AFP can be used in discrimination between HCC and ICC patients at a cut off level of $<23.6 \mathrm{ng} / \mathrm{mL}$, with $88.1 \%$ sensitivity, $61.8 \%$ specificity, $71.11 \%$ PPV and $79.6 \% \mathrm{NPV}$; area under the curve was 0.76 and $\mathrm{P}$-value was 0.002 denoting good predicative value of AFP in prediction of ICC, and this was in agreement with $\mathrm{Li}$ et al. ${ }^{28}$ who reported a AFP cut off level of $<$ $15.4 \mathrm{ng} / \mathrm{mL}$, with high sensitivity and specificity for diagnosis of CCA when combined to CA19-9.

\section{Conclusion}

CA19-9 is important in the diagnosis of the cHCC-CC in patients with typical radiological criteria of HCC.

CA19-9 and AFP are significant in diagnosis of pure cases of ICC in patients with typical radiological criteria of HCC.

Esophageal varices, jaundice, platelet count, and immuno-histochemistry are significant to differentiate between $\mathrm{HCC}$ and ICC patients. 


\section{Acknowledgments}

To all workers in NHTMRI, Benha university hospitals and to all my professors, to whom I have the honor to belong, to my professors who encouraged and supported me, who gave me their confidence and pushed me forwards.

I extend my most profound gratitude to the patients included in this study may Allah alleviate their sufferings and may all our efforts be just for their own benefit.

\section{References}

[1] A.Forner, M.Reig, J.Bruix, Hepatocellular carcinoma. Lancet, Vol.391(10127), PP.130114,2018 .

[2] T.Akinyemiju , S. Abera , M. Ahmed, N. Alam , Global Burden of Disease Liver Cancer Collaboration. The burden of primary liver cancer and underlying etiologies from 1990 to 2015 at the global, regional, and national level: Results from the Global Burden of Disease Study 2015. JAMA Oncol, Vol.3, PP.168391,2017.

[3] S.Rizvi, S.A.Khan, C.L.Hallemeier, R.K.Kelley, Cholangiocarcinoma - evolving concepts and therapeutic strategies. Nat Rev Clin Oncol, Vol.15(2), PP.95-111,2018.

[4] S.A. Kim , J.M. Lee , K.B. Lee , S.H.Kim , Intrahepatic mass-forming cholangiocarcinomas: enhancement patterns at multiphasic CT, with special emphasis on arterial enhancement pattern--correlation with clinicopathologic findings. Radiology, Vol.260(1), PP.148-57,2011.

[5] D.R.Waisberg, R.S.Pinheiro, L.S.Nacif, V.Rocha-Santos, Resection for intrahepatic cholangiocellular cancer: new advances. Transl Gastroenterol Hepatol, Vol.3, PP.60,2018.

[6] Y.I.Yoon, S .Hwang, Y.J. Lee, K.H.Kim, Postresection Outcomes of Combined Hepatocellular Carcinoma-Cholangiocarcinoma, Hepatocellular Carcinoma and Intrahepatic Cholangiocarcinoma. J Gastrointest Surg, Vol.20(2), PP.411-20,2016.

[7] K.J.Fowler, A.Sheybani, R.R.Parker, S.Doherty, Combined hepatocellular and cholangiocarcinoma (biphenotypic) tumors: imaging features and diagnostic accuracy of contrast-enhanced CT and MRI. AJR Am J Roentgenol, Vol.201(2), PP.332-9,2013.

[8] E.M.Brunt, V.Paradis, C.Sempoux, N.D.Theise, Biphenotypic (hepatobiliary) primary liver carcinomas: the work in progress. Hepat Oncol, Vol.2(3), PP.255-73,2015.

[9] M.Chi, K.Mikhitarian, C.Shi, L.W. Goff. Management of combined hepatocellularcholangiocarcinoma: a case report and literature review. Gastrointest Cancer, Vol.5, PP. 199202,2012.
[10] C.H. Wu , C.C. Yong , E.H. Liew , L.L.Tsang , Combined Hepatocellular Carcinoma and Cholangiocarcinoma: Diagnosis and Prognosis After Resection or Transplantation. Transplant Proc, Vol.48, PP.1100-4,2016.

[11] Y.H.Park , S.Hwang , C.S.Ahn , K.H.Kim , Long-term outcome of liver transplantation for combined hepatocellular carcinoma and cholangiocarcinoma. Transplant Proc, Vol.45, PP.3038-40, 2013.

[12] A.Q.Wang , Y.C.Zheng , J.Du , C.P.Zhu , Combined hepatocellular cholangiocarcinoma: Controversies to be addressed. World J Gastroenterol, Vol.22, PP.4459-65,2016.

[13] A.Sciarra, Y.N.Park, C.Sempoux, Updates in the diagnosis of combined hepatocellularcholangiocarcinoma. Hum Pathol, Vol.96, PP.48-55,2020.

[14] N.D.Theise, O.Nakashima, Y.N.Park, Y.Nakanuma,Combined hepatocellularcholangiocarcinoma. In: WHO classification of tumors of the digestive system. 4th ed. Lyon: IARC Press, 2010.

[15] S.K.Tickoo, S.Y.Zee, S.Obiekwe, H.Xiao, Combined hepatocellular-cholangiocarcinoma: a histopathologic, immunohistochemical, and in situ hybridization study. Am J Surg Pathol, Vol.26(8), PP.989-97,2002.

[16] D.H.Jung, S.Hwang, K.H.Kim, S.M.Hong, Clinicopathological Features and Post-resection Prognosis of Double Primary Hepatocellular Carcinoma and Intrahepatic Cholangiocarcinoma. World J Surg, Vol.41(3),825-834,2017.

[17] K.Wakizaka, H.Yokoo, T.Kamiyama, M.Ohira, Clinical and pathological features of combined hepatocellular-cholangiocarcinoma compared with other liver cancers. J Gastroenterol Hepatol, Vol.34(6),1074-80,2019.

[18] C.H.Lee, S.Y.Hsieh, C.J.Chang, Y.J.Lin,Comparison of clinical characteristics of combined hepatocellular-cholangiocarcinoma and other primary liver cancers. J Gastroenterol Hepatol, Vol.28(1),122-7,2013.

[19] T.Li, L.X.Qin , J.Zhou , H.C.Sun , S.J.Qiu , Q.H.Ye , Staging, prognostic factors and adjuvant therapy of intrahepatic cholangiocarcinoma after curative resection. Liver Int, Vol.34,953-60,2014.

[20] A.A. Aljumah , H. Kuriry , M .AlZunaitan , M. Al Ghobain , Clinical Presentation, Risk Factors, and Treatment Modalities of Hepatocellular Carcinoma: A Single Tertiary Care Center Experience. Gastroenterol Res Pract, PP.1989045,2016.

[21] E. Gigante , M.Ronot , C.Bertin , M.Ciolina, Combining imaging and tumor biopsy improves the diagnosis of combined hepatocellularcholangiocarcinoma. Liver Int, Vol.39, PP.2386-96,2019. 
[22] W.Y.Hsieh, P.H.Chen, I.Y. Lin , C.W.Su, The impact of esophagogastric varices on the prognosis of patients with hepatocellular carcinoma. Sci Rep, Vol.7, PP.42577,2017.

[23] W.S.Lee , K.W.Lee , J.S.Heo , S.J.Kim , Comparison of combined hepatocellular and cholangiocarcinoma with hepatocellular carcinoma and intrahepatic cholangiocarcinoma. Surg Today, Vol.36, PP.892-7,2006.

[24] F. Lumachi , G.Lo Re , R.Tozzoli , F.D'Aurizio , Measurement of serum carcinoembryonic antigen, carbohydrate antigen 19-9, cytokeratin19 fragment and matrix metalloproteinase-7 for detecting cholangiocarcinoma: a preliminary case-control study. Anticancer Res, Vol.34, PP.6663-7,2014.

[25] X.F.Zhang , T.Wei , X.M.Liu , C.Liu , Impact of cigarette smoking on outcome of hepatocellular carcinoma after surgery in patients with hepatitis B. PLoS One, Vol.9, PP.e85077,2014.

[26] G.Sapisochin , C.R.de Lope , M.Gastaca , J.O. de Urbina , al. Intrahepatic cholangiocarcinoma or mixed hepatocellular-cholangiocarcinoma in patients undergoing liver transplantation: a Spanish matched cohort multicenter study. Ann Surg, Vol.259(5), PP.944-52, 2014.

[27] H.S.Ryu , K.Lee , E.Shin , S.H.Kim , Comparative analysis of immunohistochemical markers for differential diagnosis of hepatocellular carcinoma and cholangiocarcinoma. Tumori, Vol.98, PP.478$84,2012$.

[28] Y.Li , D.J.Li , J.Chen , W.Liu , Application of Joint Detection of AFP, CA19-9, CA125 and CEA in Identification and Diagnosis of Cholangiocarcinoma. Asian Pac J Cancer Prev, Vol.16, PP.3451-5,2015 